\title{
Aqueous Solvation Dynamics at the Anionic Surfactant Air/Water Interface ${ }^{\dagger}$
}

\author{
Alexander V. Benderskii and Kenneth B. Eisenthal* \\ Department of Chemistry, Columbia University, New York, New York 10027 \\ Received: January 31, 2001
}

\begin{abstract}
Effect of the negatively charged carboxylate group of the stearic acid surfactant $\mathrm{CH}_{3}\left(\mathrm{CH}_{2}\right)_{16} \mathrm{COO}^{-}$on the solvation at the surfactant air/water interface was investigated. A direct comparison is made with the previously studied interfacial aqueous solvation at a monolayer of the neutral stearic acid $\mathrm{CH}_{3}\left(\mathrm{CH}_{2}\right)_{16} \mathrm{COOH}$, as well as the surfactant-free air/water interface. Static and time-resolved femtosecond second harmonic generation (SHG) spectroscopy of a solvatochromic dye coumarin 314 (C314) adsorbed at the interface was employed as a surface-selective molecular probe of the interfacial solvation environment. The $S_{1} \leftarrow S_{0}$ electronic transition frequency of $\mathrm{C} 314$, which reflects static solvation at the interface, is relatively insensitive to the surface charge. On the contrary, the characteristic time of the solvation dynamics at the anionic surfactant monolayer (200 $\AA^{2} /$ molecule), $\tau_{\mathrm{s}}{ }^{-}=1.6 \pm 0.3 \mathrm{ps}$, is significantly slower than at the neutral monolayer of undissociated stearic acid at the same surface coverage, $\tau_{\mathrm{s}}{ }^{0}=400 \pm 60 \mathrm{fs}$. It is also slower than the air/water interfacial solvation, with the amplitude-averaged time $\tau_{\mathrm{s}}=850 \pm 70 \mathrm{fs}$ measured without the lipid surfactant. The observed effect is consistent with the alignment of the water molecules near the interface by the electric field of the charged surfactant, which results in an increased hydrogen bond order and loss of diffusional (rotational and translational) mobility.
\end{abstract}

\section{Introduction}

Collective molecular dynamics at liquid interfaces is a fascinating subject with practical implications for many important processes in technology and life sciences. In particular, understanding of the solvation at aqueous interfaces is imperative for any predictive theory of the chemical reaction dynamics at liquid interfaces. Experimental and theoretical studies of the bulk phase solvents have shown that the solvation dynamics, which reflects dipolar relaxation of the solvent molecules in response to a charge redistribution of the solute, has a profound effect on the rates of the charge-transfer reactions. ${ }^{1-3}$ Many biologically important processes occur at aqueous interfaces, e.g., the surface of cell membranes, and often involve charge transfer as one of the steps. Reactions of folded proteins, in particular enzymes, RNA, and DNA, also require consideration of the interfacial environment of these bulky molecules, for which the term "biological water" has been coined recently. ${ }^{4}$

Tremendous complexity of the biological interfaces makes it a formidable task to analyze these systems at the molecular level. In general, however, one can classify two types of the interfacial interactions that determine the molecular structure and dynamics: (1) specific local interactions, in particular, chemical and hydrogen bonding of solvent and solute molecules with the surface moieties, and (2) nonspecific electrostatic interactions arising when ionic species are distributed on the surface. The latter can be generally thought of as interaction of charges and/or dipoles of molecules at or near the interface with the electric field of a charged surface. To understand the interfacial interactions and how they affect the solvation properties, one therefore must attempt to separate the two types.

A multitude of hydrophilic, hydrophobic, neutral, or ionized functional groups are present at a typical biological surface. Consider, for example, the headgroups of phospholipids forming

$\dagger$ Part of the special issue "Bruce Berne Festschrift". bilayer membranes or side chains of the common amino acids, with functional groups such as $-\mathrm{COOH},-\mathrm{NH}_{2},-\mathrm{OH},-\mathrm{H}$, $-\mathrm{CH}_{3}$, etc., which account for the protein folding and function of enzymes. Various combinations of these functional groups on the surface result in an entangled mix of interfacial interactions. An air/water interface covered with a chosen amphiphilic surfactant provides a simplified model system that may prove useful in an attempt to approach this complex problem. Amphiphilic surfactants such as long chain fatty acids, alcohols, amines, etc. form ordered, well-characterized monolayers at the air/water interface, ${ }^{5,6}$ with the headgroup immersed in the water phase and the organic tail pointing up in the air. 6,7 This enables one to investigate how a particular functional group (in the present study, the carboxylate/carboxyl group encountered, e.g., in the side chains of aspartic and glutamic amino acids) affects the interfacial solvation. By measuring and comparing the effect of different headgroups on solvation, one may be able to deconstruct the complex interactions at the aqueous biological interfaces into simpler components.

Many naturally occurring biological surfaces contain charged headgroups, with the compensating counterions dissolved in the adjacent aqueous phase of the buffer electrolyte solution. One example is the negatively charged cell membranes, which are composed of a mixture of phospholipids with either anionic (e.g., glycerol or serine) or zwitterionic (e.g., choline) headgroups. In many cases, for example, when carboxyl or amine groups are present at the interface, the surface charge is a function of the $\mathrm{pH}$ of the water phase. The presence of charged moieties at the interface results in a large electrostatic field imposed on both the solute and the solvent molecules in the interfacial region. For example, even for a moderate surface charge density of 1 charged group per $200 \AA^{2}$, the electric field at the interface is $\sim 7 \times 10^{7} \mathrm{~V} / \mathrm{m}$. The interaction energy of a water dipole $\mu_{\mathrm{H}_{2} \mathrm{O}}=1.85 \mathrm{D}$ with this field is of the order of $0.1 k_{\mathrm{B}} T$. It is therefore natural to expect that the surface charge of biological 
aqueous interfaces significantly affects both the structure and the dynamical properties of water near the interface.

Several experimental techniques have been employed to investigate equilibrium structural properties of water in the vicinity of charged interfaces. Using the surface-enhanced FTIR, ${ }^{8,9}$ and X-ray scattering ${ }^{10}$ for water/metal electrode surfaces, the nonlinear vibrational sum frequency generation (SFG) for air/water, organic liquid/water, and quartz/water interfaces, ${ }^{11,12}$ and $\mathrm{SHG}$ for the air/water ${ }^{13}$ and silica/water interfaces, ${ }^{14}$ alignment of the water dipoles by the interfacial electric field has been observed, along with the changes in the hydrogen bonding structure near the charged interface. A number of theoretical studies, mostly utilizing MD computer simulations, of air/water, organic liquid/water, ${ }^{15,16}$ and water/ metal electrode, ${ }^{17,18}$ interfaces have been performed in order to develop a better understanding of how the electrostatic field affects water properties in the interfacial region. One must point out, however, that the hydrogen bonded network of interacting water dipoles is a very complex system with a multitude of dynamical time scales, ${ }^{19-21}$ and predicting, even qualitatively, the electric field effect on the dynamics is a tough theoretical problem.

This paper presents a first attempt to determine how the static solvation energies and the solvation dynamics of a probe molecule at the interface, properties that directly pertain to the interfacial reaction dynamics, are influenced by the surface charge. The chosen system, which consists of a charged surfactant at the air/water interface, is designed to serve as a simplified model of the more complex biological interfaces. In the femtosecond solvation dynamics experiments, we utilize the recently developed time-resolved second harmonic generation spectroscopy, ${ }^{22-24}$ which combines surface specificity, spectroscopic selectivity, and ultrafast temporal resolution. Static electronic transition spectra and dynamics of the electronically excited-state solvation of a solvatochromic probe coumarin 314 (C314) adsorbed at the air/water interface covered with anionic stearic acid $\mathrm{CH}_{3}\left(\mathrm{CH}_{2}\right)_{16} \mathrm{COO}^{-}$are compared with the previous measurements at the neutral monolayer of the same surfactant ${ }^{24}$ and at the surfactant-free air/water interface. ${ }^{22,23}$ Both neutral and ionic monolayers of stearic acid can be readily prepared because the ionization state of the carboxyl/carboxylate functional group is determined by the $\mathrm{pH}$ of the underlying water phase. The surfactant-free air/water interface was studied previously, ${ }^{22,23}$ and two components, $\tau_{1}=250 \pm 60 \mathrm{fs}$ and $\tau_{2}=1250 \pm 80 \mathrm{fs}$ were obtained, with an amplitude-averaged solvation time of $\tau_{\mathrm{s}}=850 \pm 50 \mathrm{fs}$. These time scales are similar to the relaxation time scales observed in the bulk water ${ }^{2,19,25}$ in agreement with the predictions of the dielectric continuum theory. ${ }^{15}$ (Due to the limited time resolution, the ultrafast "inertial" component of the solvation dynamics in water, on the $<30 \mathrm{fs}$ time scale ${ }^{25}$, was not recorded in these experiments.) However, when a monolayer of neutral stearic acid $\mathrm{CH}_{3}\left(\mathrm{CH}_{2}\right)_{16}$ $\mathrm{COOH}$ was spread on the surface, the interfacial solvation time changed to $\tau_{\mathrm{s}}{ }^{0}=400 \pm 60 \mathrm{fs}^{24}$ This significant modification of the interfacial dynamics was attributed to the rearrangement of the hydrogen bonding network of water molecules near the interface due to specific interactions with the hydrophilic carboxyl group of the surfactant. ${ }^{24} \mathrm{We}$ discover that relaxation of water at the anionic carboxylate monolayer is $\tau_{\mathrm{s}}{ }^{-}=1.6 \pm$ $0.3 \mathrm{ps}$, which is significantly slower (by a factor of 4) than at the neutral carboxyl surfactant at the same surface coverage. One of the possible explanations of this effect is the nonspecific electrostatic interactions of the water dipoles with the charged plane of the carboxylate groups. Consistent with the static studies of equilibrium water structure at charged interfaces, ${ }^{8-11}$ the electrostatic field induces alignment and increased hydrogen bonding of the water molecules, thus hindering their motion.

\section{Experimental Section}

Details of the experimental apparatus and sample preparation were described in a previous publication. ${ }^{24}$ Briefly, saturated solutions of coumarin 314 (Acros, laser grade) were prepared in deionized double-distilled water at $20^{\circ} \mathrm{C}$, and the concentration was measured by UV-vis spectroscopy: the peak absorbance $A=0.65$ at $\lambda_{\max }=448 \mathrm{~nm}$ corresponding to the dye concentration $C=15 \mu \mathrm{M}$. The solution was contained in a shallow Teflon beaker, and the SHG experiments were conducted using reflection of the probe laser beam off the free surface of the solution. Surface concentration of the adsorbed C314 was $\sim 10^{13} \mathrm{~cm}^{-2}$, obtained from surface tension measurements of the $\mathrm{C} 314$ solutions. ${ }^{24}$

Langmuir monolayers of stearic acid $\mathrm{CH}_{3}\left(\mathrm{CH}_{2}\right)_{16} \mathrm{COOH}$ (Aldrich) were prepared on the surface of the sample in the beaker using hexane as a spreading solvent, which completely evaporates in less than 1 min, leaving a film of stearic acid on the water surface. The $\mathrm{pH}$ of the solution was adjusted to $\mathrm{pH}=10.5$ by adding $\mathrm{NaOH}$. The carboxyl headgroups of the amphiphilic surfactant are completely ionized because the $\mathrm{p} K_{\mathrm{a}} \approx 5.0$ of the stearic acid is well below the $\mathrm{pH}$ of the underlying solution. At the same time, it was verified by the $\mathrm{UV}$-vis spectroscopy that the $\mathrm{C} 314$ is insensitive to the solution $\mathrm{pH}$ and stable in the range from $\mathrm{pH} 3.0$ to 11.0. Surface tension measurements were performed using a Wilhelmy plate setup to determine the surface coverage corresponding to the first order 2D gas-liquid phase transition of the surfactant film. ${ }^{5,6}$ It was found that for the anionic lipid, the transition shifts to $A=280$ $\AA^{2} /$ molecule, compared to $A=260 \AA^{2} /$ molecule for the neutral form of the same surfactant. ${ }^{24}$ This is expected, due to Coulomb repulsion of the charged $-\mathrm{COO}^{-}$headgroups, which therefore require larger surface area. In the interfacial solvation dynamics experiments, we use monolayers with $A=200 \AA^{2} /$ molecule, which is the same as the neutral monolayers studied previously. ${ }^{24}$ This surface coverage corresponds to the expanded 2D liquid phase, where the film is uniform ${ }^{6}$ and orientationally ordered. ${ }^{7,26}$

A regeneratively amplified $\mathrm{Ti}-$ sapphire laser system (ClarkMXR) produces $120 \mathrm{fs}$ pulses used in the pump-probe experiment. The fundamental wavelength is tuned to $\lambda=850$ $\mathrm{nm}$, and a portion of the laser output (70 $\mu \mathrm{J} /$ pulse) was used directly as a probe beam for the second harmonic generation. A small portion of the output was frequency doubled in a BBO crystal to produce $1 \mu \mathrm{J}$ pump pulses at $425 \mathrm{~nm}$, which are onephoton resonant with the adsorbed C 314 molecules. Both pump and probe beams were directed onto the sample surface at an incidence angle $70^{\circ}$ from normal, and focused by two $1 \mathrm{~m} \mathrm{f.l}$. lenses to $0.5 \mathrm{~mm}$ spots overlapped at the surface. The sample beaker was rotated at $4 \mathrm{rpm}$ with the focal spot of the laser 1.5 $\mathrm{cm}$ off center to avoid local heating, desorption, and bleaching of the C314 dye by the one-photon resonant pump beam. The pump and probe beams were recombined at a $5^{\circ}$ angle in the horizontal plane, and the reflected beams were spatially separated after the sample using 4 irises. A long-pass wavelength colored glass filter was placed in the probe beam immediately before the sample to block any spurious $\mathrm{SH}$, and a short-pass blue filter was placed immediately after the sample surface to block the reflected fundamental wavelength. The generated $\mathrm{SH}$ beam was dispersed through a $1 / 4 \mathrm{~m}$ monochromator (Jarrel Ash) and detected by a Hamamatsu PMT (model R4220P). The signal was boxcar integrated, averaged (Stanford Research 
TABLE 1: Properties of the Molecular Probe Coumarin 314 Adsorbed at the Various Air/Water Interfaces

\begin{tabular}{lccc}
\hline & \multicolumn{2}{c}{ Interface } \\
\cline { 2 - 4 } & negatively charged & neutral & \\
& $\mathrm{CH}_{3}\left(\mathrm{CH}_{2}\right)_{16} \mathrm{COO}^{-}$, & $\mathrm{CH}_{3}\left(\mathrm{CH}_{2}\right)_{16} \mathrm{COOH}$, & \\
& $A=200 \AA^{2} /$ molecule $^{a}$ & $A=200 \AA^{2} /$ molecule $^{b}$ & surfactant-free air/water interface $^{c}$ \\
\hline molecular angle (wrt surface normal) $\theta_{\text {mol }}$ & $30 \pm 4^{\circ}$ & $41 \pm 3^{\circ}$ & $70 \pm 3^{\circ}$ \\
$\mathrm{S}_{1} \leftarrow \mathrm{S}_{0}$ transition freq $\omega_{\mathrm{ge}}$ & $431 \pm 2 \mathrm{~nm}$ & $432 \pm 2 \mathrm{~nm}$ & $423 \pm 2 \mathrm{~nm}$ \\
$\mathrm{~S}_{1} \leftarrow \mathrm{S}_{0}$ transition width $\Gamma_{\text {ge }}$ & $10 \pm 2 \mathrm{~nm}$ & $19 \pm 2 \mathrm{~nm}$ & $14 \pm 2 \mathrm{~nm}$ \\
solvation time $\tau_{\mathrm{s}}$ & $1.6 \pm 0.3 \mathrm{ps}$ & $400 \pm 60 \mathrm{fs}$ & $250 \pm 60 \mathrm{fs} ; 1250 \pm 80 \mathrm{fs} \tau_{\text {avg }}=850 \pm 70 \mathrm{fs}$
\end{tabular}

${ }^{a}$ This study. ${ }^{b}$ From ref $24 .{ }^{c}$ From refs 22, 23, 24.

Systems), and recorded by a computer (National Instruments A/D board), which controlled the delay between the pump and probe pulses via a stepper-motor driven translational stage (Klinger).

\section{Results and Discussion}

We begin by describing the static SHG spectroscopy of the C314 probe molecule at the negatively charged stearic acid monolayer. The surface charge density of the $200 \AA^{2} /$ molecule anionic monolayer is $-8 \mu \mathrm{C} / \mathrm{cm}^{2}$. The results are summarized in Table 1. Molecular orientation of the probe dye was measured using the null angle technique,${ }^{27}$ assuming a narrow distribution of the molecular angles. At $A=200 \AA^{2} /$ molecule of anionic stearic acid, the average angle between the transition dipole moment of C314 and the interface normal is $\theta_{\text {mol }}=30 \pm 5^{\circ}$, whereas in the case of neutral surfactant monolayer of the same density, $\theta_{\text {mol }}=41 \pm 3^{\circ} .{ }^{24}$ The $\mathrm{S}_{1} \leftarrow \mathrm{S}_{0}$ transition dipole moment is parallel to the permanent dipole moment of $\mathrm{C} 314$ in the ground electronic state. The observed effect, $\Delta \theta_{\mathrm{mol}} \sim 10^{\circ}$, is therefore consistent with the alignment of the polar C314 molecules $\left(\mu_{0}=8.2 \mathrm{D}^{28}\right)$ along the electrostatic field of the carboxylate monolayer $\left(E=6.5 \times 10^{7} \mathrm{~V} / \mathrm{m}\right)$, which is normal to the interface. The field-dipole interaction energy can be estimated to be of the order of $k_{\mathrm{B}} T$.

Spectra of the C314 adsorbed at the interface were measured by recording the intensity of the second harmonic signal while tuning the fundamental wavelength of the probe laser beam (see refs 29 and 30 for details of the SH spectroscopy). Two-photon resonant enhancement is observed when the SH frequency $2 \omega_{\mathrm{pr}}$ of the Ti-sapphire laser output (wavelength tuning range 800$900 \mathrm{~nm}$ ) matches the $\mathrm{S}_{1} \leftarrow \mathrm{S}_{0}$ electronic transition frequency $\omega_{\mathrm{ge}}$ (ground-to-excited) of the C314 (usually in the 420-450 nm range). Nonresonant second harmonic signal from a neat silica/ water interface was recorded as a reference for normalization to take into account laser intensity variation as the wavelength is tuned. The $\mathrm{SH}$ spectrum of $\mathrm{C} 314$ at the interface covered with the charged stearic acid monolayer (Figure 1) shows a peak close to that of the neutral surfactant. The asymmetry in the SH spectrum arises due to the nonresonant contribution to the SH signal from water molecules that are partially ordered near the interface by the electric field of the monolayer. ${ }^{14}$ The solid line in Figure 1 shows the fit to a model, which takes this nonresonant contribution into account: ${ }^{31}$

$$
I_{\mathrm{SH}} \propto\left|\frac{A_{\mathrm{ge}}}{\omega_{\mathrm{ge}}-2 \omega_{\mathrm{pr}}+i \Gamma_{\mathrm{ge}}}+B\right|^{2}
$$

with the adjustable parameters $A_{\mathrm{ge}}, B, \omega_{\mathrm{ge}}$, and $\Gamma_{\mathrm{ge}}$. The factor $A_{\text {ge }}$ contains the number of $\mathrm{C} 314$ probe molecules, the transition matrix elements, and other terms that are not strongly dependent on the probe frequency $\omega_{\mathrm{pr}}$. Due to the nonresonant term $B$, the apparent maximum of the $\mathrm{SH}$ spectrum is slightly blue-shifted

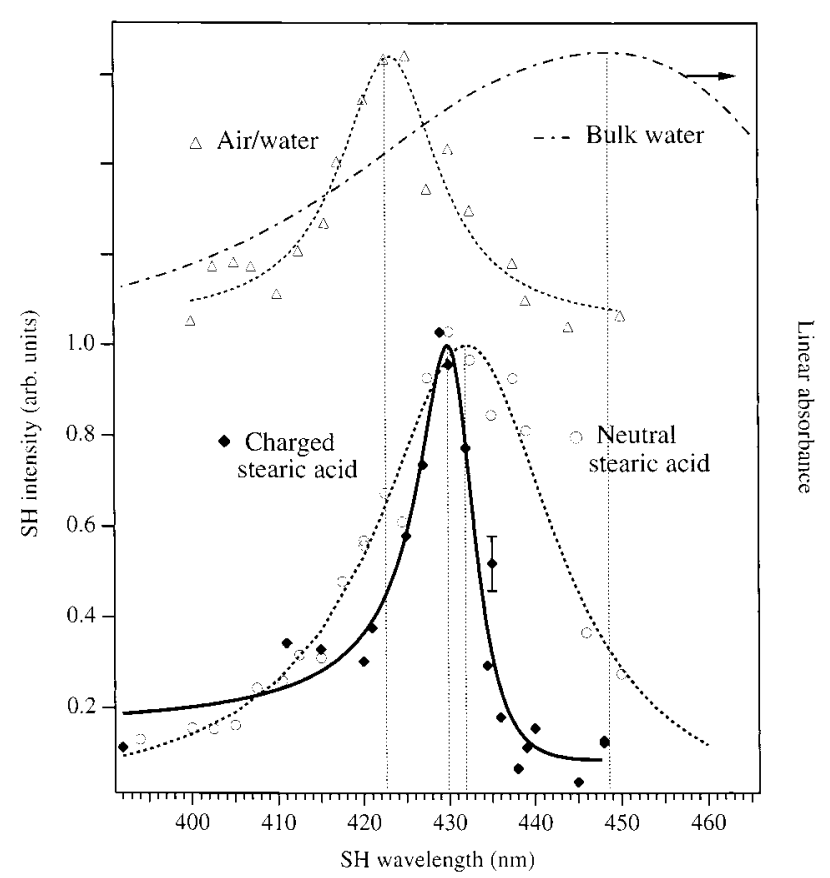

Figure 1. Lower traces: second harmonic spectrum of coumarin 314 adsorbed at the air-water interface covered with a negatively charged stearic acid, $A=200 \AA^{2} /$ molecule, solid line shows the fit to eq 1 , see text for details. $\bigcirc$, dashed line - same for the neutral stearic acid monolayer, $A=200 \AA^{2} /$ molecule, ref 24 . Upper traces: $\Delta$, dashed line - SH spectrum of $\mathrm{C} 314$ at the surfactant-free air/water interface, ref 24; dash-dotted line - linear absorption spectrum of C314 in bulk water.

from the real $\mathrm{S}_{1} \leftarrow \mathrm{S}_{0}$ transition frequency $\omega_{\mathrm{ge}}$ of C314 by $\sim 2$ $\mathrm{nm}$. With this correction taken into account, the transition frequency $\omega_{\text {ge }}$ of $\mathrm{C} 314$ at the charged stearic acid monolayer $(431 \pm 2 \mathrm{~nm})$ is quite close to $\omega_{\text {ge }}$ at the neutral monolayer $\left(432 \pm 2 \mathrm{~nm},{ }^{24}\right)$. Note that the interfacial electrostatic field in our experiments is too weak to induce a significant electrochromic shift of the transition frequency: the transition dipole moment of $\mathrm{C} 314$ is approximately $\mu_{\mathrm{ge}}=4 \mathrm{D}$ while $E=6.5 \times$ $10^{7} \mathrm{~V} / \mathrm{m}$, and we estimate the electrochromic shift to be less than $50 \mathrm{~cm}^{-1}$, i.e., $1 \mathrm{~nm}$. The shift of the electronic transition frequency of the solvatochromic probe such as $\mathrm{C} 314$ reflects the polarity of the solvation environment. ${ }^{31} \mathrm{We}$ therefore conclude that the static polarity at the air/water interface covered with negatively charged stearic acid monolayer is similar to that experienced by the probe molecule at the neutral monolayer and somewhere between the polarities of the bulk water and the surfactant-free air/water interface (Figure 1). Using the ET(30) scale to compare the polarity of interfaces and the bulk media, ${ }^{31}$ the negatively charged stearic acid monolayer at the air/water interface approximately corresponds to the polarity of bulk chlorobenzene, $\operatorname{ET}(30)=37.5$. For comparison, the surfactant-free air/water interface has $\operatorname{ET}(30)=31$, close to that of bulk hexane, while for the bulk water, ET(30) $=63$. 
Interfacial solvation dynamics were measured in a femtosecond pump-probe experiment in the spirit of the numerous bulk-phase solvation dynamics experiments: ${ }^{2,19,25}$ the dye molecule is electronically excited by a pump laser pulse, and the subsequent solvent relaxation is monitored by a time-delayed probe pulse as the energy gap between the excited and the ground states $\mathrm{S}_{1} \rightarrow \mathrm{S}_{0}$ evolves in time. Unlike the time-resolved fluorescence experiments used in the bulk phase solvation studies, where only the excited $\mathrm{S}_{1}$ state fluoresces, the $\mathrm{SH}$ signal from the interface contains resonant contributions from both ground and excited-state populations. The excited-state nonlinear susceptibility evolves in time (increases or decreases) as solvation brings the $\mathrm{S}_{1} \rightarrow \mathrm{S}_{0}$ energy gap $\omega_{\text {eg }}(t)$ (excited-to-ground) for the excited-state molecules in or out of the resonance with the $\mathrm{SH}$ frequency $2 \omega_{\mathrm{pr}}$. The ground state contribution is timeindependent. Assuming a two-level model (i.e., only two electronic states resonantly contribute to the SHG),

$$
\begin{aligned}
& I_{\mathrm{SH}}(t) \propto \\
& \left|n_{\mathrm{g}} \frac{A_{\mathrm{ge}}}{\omega_{\mathrm{ge}}-2 \omega_{\mathrm{pr}}+i \Gamma_{\mathrm{ge}}}+n_{\mathrm{e}} \frac{A_{\mathrm{eg}}}{\omega_{\mathrm{eg}}(t)-2 \omega_{\mathrm{pr}}+i \Gamma_{\mathrm{eg}}}+B\right|^{2}
\end{aligned}
$$

where the first term describes the ground-state resonant contribution $\chi^{(2)}$, the second term is the excited-state resonant contribution $\chi^{(2)}$, and the third term is nonresonant nonlinear susceptibility $\chi^{(2)} \mathrm{NR} ; n_{\mathrm{g}}$ and $n_{\mathrm{e}}$ represent fractions of the C314 molecules in the ground and excited states, $n_{\mathrm{g}}+n_{\mathrm{e}}=1$. Because the second harmonic generation is a coherent optical process, the three contributions interfere constructively or destructively, depending on their relative phases, which is reflected by the complex values of their respective nonlinear susceptibilities in eq 2 .

Upon the initial excitation of the $\mathrm{S}_{1}$ state, before the solvation takes place, $\omega_{\text {eg }}(0)$ is close to $\omega_{\text {ge. }}$. Because $A^{\prime}{ }_{\text {eg }}=-A_{\text {ge }}^{\prime}{ }^{30,22}$ an initial decrease in the $\mathrm{SH}$ intensity as a function of the pump-probe delay is observed when the pump and probe pulses overlap in time. Upon photoexcitation, the ground-state contribution is reduced by a fraction $n_{\mathrm{e}} / n_{\mathrm{g}}$, and the amplitude of the excited state contribution is approximately $n_{\mathrm{e}} / n_{\mathrm{g}}$ of the ground state, but with an opposite sign. This makes the initial decrease of the SH signal proportional to $2 n_{\mathrm{e}} / n_{\mathrm{g}}$, eq 2 . Thus the instrument response limited $\sim 10 \%$ drop in the SH signal at $t=$ 0 in Figure 2 corresponds to $\sim 5 \%$ of the $\mathrm{C} 314$ molecules being promoted to the $S_{1}$ state by the pump pulse. Because the chosen probe wavelength $\left(\lambda_{\mathrm{SH}}=425 \mathrm{~nm}\right)$ is on the blue side of the $\mathrm{S}_{1} \leftarrow \mathrm{S}_{0}$ transition at this interface (Figure 1), the solvation lowers the excited-state energy and shifts $\omega_{\text {eg }}(\mathrm{t})$ to the red, away from the resonance with the probe. The second term in eq 2 therefore decreases in magnitude, thereby reducing the destructive interference, and the $\mathrm{SH}$ signal shows a partial recovery (Figure 2).

The measured $I_{\mathrm{SH}}(\mathrm{t})$ dependence was fit to the two-level model outlined above by assuming a single-exponential decay for the solvation time correlation function ${ }^{2}$

$$
S(t)=\frac{\omega_{\mathrm{eg}}(t)-\omega_{\mathrm{eg}}(\infty)}{\omega_{\mathrm{eg}}(0)-\omega_{\mathrm{eg}}(\infty)}=\exp \left(-t / \tau_{\mathrm{S}}\right)
$$

and substituting the resulting $\omega_{\text {eg }}(t)$ into eq 2 . The pump-probe cross-correlation function (fwhm $\approx 175 \mathrm{fs}$ ) was measured independently using their sum frequency generation at the interface and was convoluted with eq 2 to correctly fit the initial drop. $A_{\text {eg }}^{\prime}, n_{\mathrm{e}} / n_{\mathrm{g}}, \tau_{\mathrm{s}}$, and $\omega_{\mathrm{eg}}(\infty)$ are adjustable parameters, while $\omega_{\text {ge }}, \Gamma_{\text {ge }}$, and $B$ are obtained by fitting the independently measured SH spectrum (Figure 1 ), and we assume $\Gamma_{\text {eg }} \approx \Gamma_{\text {ge }}$.

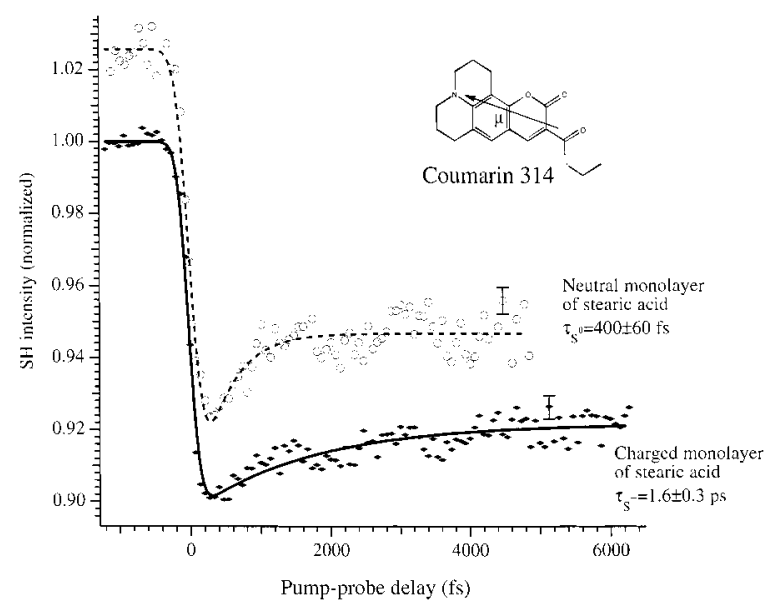

Figure 2. Lower trace: $\bullet$ time-resolved second harmonic signal recorded in a pump-probe solvation dynamics experiment of C314 at the air-water interface covered with a negatively charged stearic acid, $A=200 \AA^{2} /$ molecule; solid line - fit to eq 2 , see text for details. Upper trace: $\bigcirc$, dashed line - same for the C 314 at the neutral stearic acid monolayer, $A=200 \AA^{2} /$ molecule, ref 24 .

The extracted solvation time for the air/water interface covered with negatively charged stearic acid is $\tau_{\mathrm{s}}{ }^{-}=1.6 \pm 0.3 \mathrm{ps}$. Use of a double exponential did not result in a better fit of the experimental data within the signal-to-noise ratio.

The upper trace in Figure 2 shows the solvation dynamics measured at the neutral stearic acid monolayer, ${ }^{24}$ where similar analysis yields the characteristic solvation time $\tau_{\mathrm{s}}{ }^{0}=400 \pm 60$ fs. Thus, it appears that the negatively charged carboxylate groups at the air/water interface induce slower aqueous relaxation than the neutral carboxyl groups. It is also interesting to compare the results at the anionic stearic acid monolayer interface with the two solvation components, $\tau_{1}=250 \pm 60 \mathrm{fs}$ and $\tau_{2}=1250 \pm 80 \mathrm{fs}$, measured at the neat air/water interface without the lipid surfactant. ${ }^{23}$ The amplitude-averaged solvation time at the neat air/water interface is $\tau_{\mathrm{s}}=850 \pm 70 \mathrm{fs}$. The two solvation time scales at the air/water interface are similar to those measured in the bulk water for similar probe dyes: coumarin 343 yielded $\tau_{1}=250 \mathrm{fs}$ and $\tau_{2}=960 \mathrm{fs}$, ${ }^{32}$ whereas 7-dimethylaminocoumarin-4-acetate yielded $\tau_{1}=160 \mathrm{fs}$ and $\tau_{2}=1.2$ ps. $^{33}$

Motions of water molecules contributing to the solvation dynamics can be divided into two categories. ${ }^{19}$ The inertial component of solvation, which constitutes as much as $50 \%$ of the overall solvation response in the bulk water, ${ }^{25}$ is associated with the nearly free rotation of the $\mathrm{H}_{2} \mathrm{O}$ dipoles. ${ }^{20}$ This component has a Gaussian time dependence and proceeds on the ultrafast time scale, $\leq 30 \mathrm{fs}$ in the bulk, ${ }^{25}$ which is beyond the temporal resolution of our experiment. The exponential solvation dynamics component observed in our measurements is referred to as the diffusive component ${ }^{2,19,25}$ and is generally associated with diffusive (overdamped) reorientational and translational motions of the water molecules. Theoretical models and molecular dynamics simulations show that these diffusive dynamics are accompanied by breaking and formation of the hydrogen bonds between water molecules. ${ }^{21}$ One may speculate, therefore, that the time scales of the diffusive solvation dynamics, such as those in Figure 2, depend on the structure and hydrogen bond order of water in the interfacial region.

A thorough theoretical modeling involving accurate carboxylate-water and carboxyl-water interaction potentials and MD simulations would be required for an unambiguous interpretation of the observed carboxylate/carboxyl surfactant effect on the solvation dynamics, which is outside of the scope of this paper. 
Here, we discuss only the possible mechanisms for the observed difference between the solvation dynamics at the carboxylate and carboxyl surfactant interfaces. Due to its negative charge, the carboxylate surfactant imposes a strong electrostatic field on the water molecules in the interfacial region. The nonspecific electrostatic interactions may alter the water structure and, therefore, the solvation dynamics time scale. On the other hand, different chemical makeup of the carboxylate vs carboxyl headgroups may lead to the different specific local interactions at the surfactant interfaces, e.g., different hydrogen bonding of the water molecules with the headgroups. This could also lead to rearrangement of the network of water-water hydrogen bonds near the interface and therefore cause the change in the dynamical response. This mechanism involving the specific local interactions was proposed in our previous work to explain the difference in the solvation dynamics observed at the neutral stearic acid monolayer vs the surfactant-free air/water interface. ${ }^{24}$

Numerous experimental studies supported by theoretical models provide evidence that the equilibrium water structure near a charged surface is significantly affected by the interfacial electrostatic field. Alignment of water molecules by the electric field was observed at metal electrodes using surface FTIR $^{8,9}$ and X-ray scattering, ${ }^{10}$ at the surface charge densities similar to our experiment, of the order of $-10 \mu \mathrm{C} / \mathrm{cm}^{2}$. Significant perturbation of the hydrogen bond network near the surface by the applied electric field was inferred from the experimental data. Using the nonresonant second harmonic generation, net orientation of water molecules was observed at a charged monolayer air/water interface ${ }^{13}$ and a charged silica surface. ${ }^{14}$ Vibrational sum-frequency generation (SFG) spectroscopy has been applied to study structure and hydrogen bonding at various aqueous interfaces. ${ }^{11,12}$ At charged surfaces, both alignment and increase in the H-bond order were observed via an overall increase in the intensity of the SFG-active $\mathrm{OH}$ stretch modes and a change in the relative intensities of the subbands assigned to weaker $\mathrm{H}$-bonded water molecules (bulk-like environment) and stronger $\mathrm{H}$-bonded molecules (ice-like structure). Perhaps the closest example to our experimental system was the vibrational SFG study of a negatively charged surfactant (sodium dodecyl sulfate) monolayer at the air/water interface, ${ }^{12}$ where the effect was observed at the surfactant coverages $A=100-$ $300 \AA^{2} /$ molecule (surface charge densities -4 to $-12 \mu \mathrm{C} / \mathrm{cm}^{2}$ ), which is very similar to our case. The observed increase of the stronger $\mathrm{H}$-bonded ice-like $\mathrm{OH}$ band with the (negative) surface charge indicates increased hydrogen bond order of water molecules near the anionic surfactant interface. In relation to our experiments, the stronger $\mathrm{H}$-bonded water structure near the interface would imply slower diffusive orientational and translational relaxation, which is associated with the rearrangement, i.e., breaking, of the water-water hydrogen bonds.

Computer simulations have been performed by several groups in an attempt to gain some insights into the molecular structure and properties of water near charged interfaces. ${ }^{16-18}$ Most of the studies, however, have focused on metal surfaces. ${ }^{17,18}$ Although all computer models reliably reproduce the water dipole alignment effect, difficulties exist in correctly describing the rearrangement of the hydrogen bond network in the electric field. An attempt to predict the electric field effect on the structure and dynamics at the dichloroethane/water interface from molecular dynamics simulations was made. ${ }^{16}$ However, similar to the other MD studies, no significant changes in the hydrogen bonding pattern were discovered, and the calculated water dipole reorientation correlation function was found to be insensitive to the applied electric field. Several reasons prevent direct correlation of the MD results of ref 16 with our experimental findings. The dichloroethane/water interface may have a very different molecular structure and therefore different dynamical properties than the surfactant air/water interface. This was found to be the case in the vibrational SFG study of the $\mathrm{CCl}_{4} /$ water and air/water interfaces. ${ }^{12}$ The comparison between the calculated water dipole reorientation correlation function and our experimental observable, the solvation correlation function $S(t)$ ( eq 3), may not be straightforward as different motions of water molecules may contribute differently to the solvation energy. ${ }^{19,20,25}$ Finally, the question remains open whether the observed difference between the carboxylate and carboxyl surfactants can be contributed entirely to the surface charge effect, or if there is an interplay between the electric field effect and the difference in the specific local interactions of water with carboxylate vs carboxyl headgroups. Further experiments such as comparative studies of the solvation dynamics at different surfactant monolayers are needed to characterize the effects of the chemical composition of the headgroup vs charge on the interfacial solvation.

\section{Conclusions}

Static and dynamic solvation of a probe molecule coumarin 314 at the air/water interface covered with an anionic carboxylate surfactant (stearic acid) was characterized and compared with the neutral surfactant air/water interface. The static solvation energetics appears to be relatively insensitive to the ionization state of the stearic acid surfactant. Solvation dynamics at the negatively charged carboxylate monolayer was found to be significantly slower than at the neutral carboxyl surfactant, $\tau_{\mathrm{s}}{ }^{-}=1.6 \pm 0.3 \mathrm{ps}$ vs $\tau_{\mathrm{s}}{ }^{0}=400 \pm 60 \mathrm{fs}$. The effect is consistent with the alignment and increased hydrogen bond order of water molecules by the electric field of the charged interface, which causes slower diffusive relaxation associated with breaking of the water-water hydrogen bonds. However, it is possible that both the nonspecific electrostatic interactions and the specific local interactions between water and carboxylate vs carboxyl headgroups of the surfactant contribute to the observed effect. Our results indicate that the dynamics of many reactions at interfaces such as electron transfer, which are controlled by the relaxation properties of the interfacial solvent environment, may be sensitive to the ionization state of the chemical moieties composing the interface.

Acknowledgment. The authors gratefully acknowledge the Division of Chemical Science, Office of Basic Energy Sciences, the Department of Energy, and the National Science Foundation for their support. We also wish to thank Mr. Joel Henzie for his contributions to this research project.

\section{References and Notes}

(1) Heitele, H. Angew. Chem., Int. Ed. Engl. 1993, 32, 359.

(2) Barbara, P. F.; Jarzeba, W. Adv. Photochem. 1990, 15, 1.

(3) Voth, G. A.; Hochstrasser, R. M. J. Chem. Phys. 1996, 100, 13034.

(4) Nandi, N.; Bagchi, B. J. Phys. Chem. B 1997, 101, 10954. Nandi, N.; Bhattacharyya, K.; Bagchi, B. Chem. Rev. 2000, 100, 2013.

(5) Adamson, A. W. Physical Chemistry of Interfaces; Wiley-Interscience: New York, 1982.

(6) Gaines, G. L., Jr. Insoluble Monolayers at Liquid-Gas Interfaces; Wiley-Interscience: New York, 1966.

(7) Guyot-Sionnest, P.; Hunt, J. H.; Shen, Y. R. Phys. Rev. Lett. 1987, $59,1597$.

(8) Habib, M. A.; Bockris, J. O’M. Langmuir 1986, 2, 388. 10664 
(10) Toney, M. F.; Howard, J. N.; Richer, J.; Borges, G. L.; Gordon, J. G.; Melroy, O. R.; Wiesler, D. G.; Yee, D.; Sorensen, L. B. Nature 1994 368, 444. Gordon, J. G.; Melroy, O. R.; Toney, M. F. Electrochim. Acta $1995,40,3$.

(11) Du, Q.; Superfine, R.; Freysz, E.; Shen, Y. R. Phys. Rev. Lett. 1993, 70, 2313. Du, Q.; Freysz, E.; Shen, Y. R. Science, 1994, 264, 826. Du, Q.; Freysz, E.; Shen, Y. R. Phys. Rev. Lett. 1994, 72, 238.

(12) Gragson D. E.; Richmond, G. L. J. Am. Chem. Soc. 1998, 120, 366. Gragson D. E.; Richmond, G. L. J. Phys. Chem. B 1998, 102, 3847. 513.

(13) Ong, S.; Zhao, X.; Eisenthal, K. B. Chem. Phys. Lett. 1993, 202,

(14) Ong, S.; Zhao, X.; Eisenthal, K. B. Chem. Phys. Lett. 1992, 191, 327

(15) Benjamin, I. Chem. Rev. 1996, 96, 1449.

(16) Schweighofer, K. J.; Benjamin, I. J. Electroanal. Chem. 1995, 391,

(17) Schweighofer, K. J.; Xia, X.; Berkowitz, M. L. Langmuir 1996, 12, 3747. Xia, X.; Berkowitz, M. L. Phys. Rev. Lett. 1995, 74, 3193. Xia, X.; Perera, L.; Essmann, U.; Berkowitz, M. L. Surf. Sci. 1995, 335, 401

(18) Nagy, G.; Heinzinger, K.; Spohr, E. Faraday Discuss. 1992, 94, 307. Nagy, G.; Heinzinger, K. J. Electroanal. Chem. 1990, 296, 549.

(19) Maroncelli, M. J. Mol. Liquids 1993, 57, 1

(20) Maroncelli, M.; Kumar, V. P.; Papazyan, A. J. Phys. Chem. 1993, 97,13

(21) Luzar, A.; Chandler, D. Nature 1996, 379, 55 and references therein.
(22) Zimdars, D.; Dadap, J. I.; Eisenthal, K. B.; Heinz, T. F. Chem Phys. Lett. 1999, 301, 112. Zimdars, D.; Eisenthal, K. B. J. Phys. Chem. A 1999, 103, 10567.

(23) Zimdars, D.; Eisenthal, K. B. J. Phys. Chem. B 2001, 105, 3993.

(24) Benderskii, A. V.; Eisenthal, K. B. J. Phys. Chem. B 2000, 104, 11723

(25) Jimenez, R.; Fleming, G. R.; Kumar, P. V.; Maroncelli, M. Nature 1994, 369, 471 .

(26) Zhang, D.; Gutow, J.; Eisenthal, K. B. J. Phys. Chem. 1994, 98, 13729.

(27) Heinz, T. F.; Tom, H. W. K.; Shen, Y. R. Phys. Rev. A 1983, 28, 1883.

(28) Moylan, C. R. J. Phys. Chem. 1994, 98, 13513.

(29) Heinz, T. F.; Chen, C. K.; Ricard, D.; Shen, Y. R. Applied Phys. $B$ 1982, 28, 229.

(30) Shen, Y. R. The Principles of Nonlinear Optics, Wiley-Interscience: New York, 1984.

(31) Wang, H.; Borguet, E.; Eisenthal, K. B. J. Phys. Chem. A 1997 101, 713. Wang, H.; Borguet, E.; Eisenthal, K. B. J. Phys. Chem. B 1998, $102,4927$.

(32) Walker, G. C.; Jarzeba, W.; Kang, T. J.; Johnson, A. E.; Barbara, P. F. J. Opt. Soc. Am. B 1990, 7, 1521.

(33) Jarzeba, W.; Walker, G. C.; Johnson, A. E.; Kahlow, M. A.; Barbara, P. F. J. Chem. Phys. 1998, 92, 7039. 